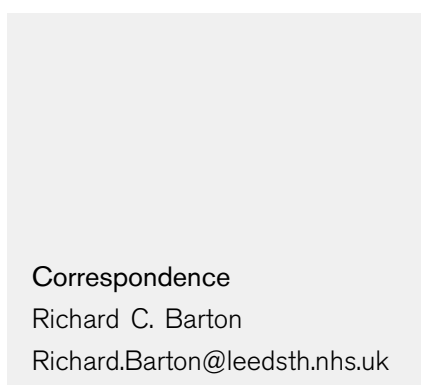

Received 14 December 2005

Accepted 13 July 2006

\section{Arthroconidia production in Trichophyton rubrum and a new ex vivo model of onychomycosis}

\author{
S. Amir Yazdanparast ${ }^{1} \dagger$ and Richard C. Barton ${ }^{2}$ \\ ${ }^{1}$ Department of Microbiology, University of Leeds, Leeds LS2 9JT, UK \\ ${ }^{2}$ Mycology Reference Centre, Department of Microbiology, Leeds General Infirmary, Leeds LS1 \\ 3EX, UK
}

\begin{abstract}
The dermatophyte fungus Trichophyton rubrum often produces arthroconidia in vivo, and these cells are thought to be involved in pathogenesis, and, in shed skin scales, to act as a source of infection. The purpose of this study was (i) to examine the environmental and iatrogenic factors which affect arthroconidiation in T. rubrum in vitro, (ii) to look at arthroconidia formation in a large number of clinical isolates of $T$. rubrum and (iii) to develop a new model for the study of arthroconidia formation in nail tissue. Arthroconidia production was studied in T. rubrum grown on a number of media and under conditions of varying $\mathrm{pH}$, temperature and $\mathrm{CO}_{2}$ concentration. The effect of the presence of antifungals and steroids on arthroconidia formation was also examined. Nail powder from the healthy toenails of volunteers was used as a substrate for arthroconidial production. On Sabouraud dextrose agar in the presence of $10 \% \mathrm{CO}_{2}$ plus air, arthroconidial formation occurred optimally at $37^{\circ} \mathrm{C}$ and $\mathrm{pH} 7 \cdot 5$, and was maximal at 10 days. Most isolates of T. rubrum showed a similar level of arthroconidial production, and only two out of 50 strains were unable to produce arthroconidia. Subinhibitory levels of some antifungals and betamethasone resulted in the stimulation of arthroconidia formation. Arthroconidial production in ground nail material also occurred under the same optimal conditions, but took longer to reach maximal levels (14 days). These in vitro and ex vivo results provide a useful basis for the understanding of arthroconidium formation in vivo in infected tissues such as nails.
\end{abstract}

\section{INTRODUCTION}

The high incidence of dermatophyte infections makes them an important public health problem (Aly, 1994), and studies estimate that between 10 and $15 \%$ of the population worldwide is affected (Matsumoto, 1996). In spite of rapid improvements in treatment during the past decade, many aspects of dermatophyte infections remain poorly understood. Dermatophyte infection of the nail, known as tinea unguium or onychomycosis, is perhaps the most refractory to treatment, and requires several months of systemic therapy, with terbinafine being the most effective agent (Evans, 1999), even though a success rate of greater than $80-85 \%$ cannot be expected. Possible reasons for the $20 \%$ failure rate include the development of antifungal resistance, poor pharmacokinetics due to the characteristics of the nails (i.e. excessive thickness) and the presence of resistant structures such as subungual dermatophytomas (Evans \& Roberts, 1998), and arthroconidia, which are frequently seen in infected nails, and whose role in disease has long been debated (Chin \& Knight, 1957). Arthroconidium formation, characteristic of dermatophyte infection of skin, hair or

tPresent address: Department of Mycology, Iran University of Medical Sciences, PO Box 15875-6171, Tehran, Iran. nails, results from the fragmentation of a hypha following multiple septum formation. Growth in the stratum corneum of skin, nails and hair occurs in the form of hyphae, which may then form arthroconidia. It has been suggested that arthroconidia are more commonly found in wet skin lesions and nails (Miyazi et al., 1971). The potential of arthroconidia to contribute to resistance to treatment is suggested by the fact that having no immediate exogenous nutritional requirements, they are resistant to adverse environmental conditions (Hashimoto \& Blumenthal, 1978). Arthroconidia shed from lesions are likely to be the form involved in the spread of infection. The development of arthroconidia in Trichophyton mentagrophytes has been observed in vitro by a number of authors (Bibel et al., 1979; Emyanitoff \& Hashimoto, 1979; Weigl \& Hejtmanek, 1979; Wright et al., 1984) and in Trichophyton rubrum (Miyaji \& Fujiwara, 1971). Here we describe a detailed examination of the environmental and iatrogenic factors which affect arthroconidiation in vitro in T. rubrum, and describe a new ex vivo model for the study of arthroconidium formation in nails.

\section{METHODS}

Organism. A total of 50 strains of T. rubrum, isolated from the skin 
and nails of patients, were used. Cultures were maintained at $27^{\circ} \mathrm{C}$ by monthly subculture on Sabouraud dextrose agar (SDA), and permanent cultures were stored at $-70{ }^{\circ} \mathrm{C}$ in $1.6 \%$ DMSO.

Media and culture conditions. For arthroconidium formation, a standard $\mathrm{pH}$ of 5.6 was employed for SDA (Oxoid); other pHs were obtained by the addition of sodium hydroxide. When medium without glucose was required, peptone agar (Difco) at 1,3, 5 and $10 \%$ was used. Brain heart infusion (BHI, Oxoid), Trichophyton no. 1 (Difco), and SDA plus 1, 3, 5 and $10 \% \mathrm{NaCl}$, were also used. Betamethasone 17-valerate, amphotericin B, itraconazole, griseofulvin and terbinafine were added to SDA to investigate their effects on arthroconidium formation. Each antifungal was dissolved in DMSO, and DMSO at the same concentration was used as a control. Cultures were incubated for up to 10 days at various temperatures, either under aerobic conditions or in air plus various $\mathrm{CO}_{2}$ concentrations using a LEEC $\mathrm{CO}_{2}$ incubator in which the $\mathrm{CO}_{2}$ concentration was set and controlled automatically by the incubator.

Microscopic examination. Adhesive tape was cut in to $1 \mathrm{~cm}$ squares, and was placed with sterile forceps on the surface of a colony, lifted off, and mounted colony side up, and a drop of $70 \%$ ethanol and a drop of lactophenol cotton blue were placed on top. The number of arthroconidia was determined after examining four randomly selected fields of view under $\times 400$ magnification. Arthroconidia were defined as any hyphal compartment $<4 \mu \mathrm{m}$ long or any spherical cell representing a disarticulated arthroconidium that was not obviously an aleuroconidium (microconidium).

Ground nails for ex vivo experiment. Clippings from several healthy toenails previously found free from fungi when examined in $10 \% \mathrm{KOH}$ were cleaned with $70 \%$ ethanol, dried in a sterile Petri dish at $37^{\circ} \mathrm{C}$, and ground with liquid nitrogen to a powder with a pestle and mortar. Plates of T. rubrum on SDA grown aerobically were harvested at 2 weeks by gently scraping the surface of the agar with a glass rod. The cells were suspended in distilled water, the $\mathrm{pH}$ was adjusted to $7 \cdot 5$ with $\mathrm{NaOH}$, and the concentration of microco-

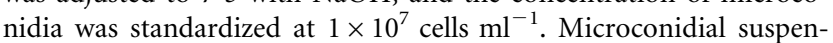
sion of T. rubrum $(20 \mu \mathrm{l})$ was spread over the surface of $0 \cdot 2 \mathrm{~g}$ nail powder on a sterile microscope slide, and the inoculated slide placed on a glass rod within a sterile glass Petri dish. Sterile water was added to the Petri dish to maintain a humid atmosphere, and then incubatation was carried out aerobically or in $10 \% \mathrm{CO}_{2}$ at $37^{\circ} \mathrm{C}$ for 14 days. The powder was then mounted, and examined microscopically in $10 \% \mathrm{KOH}$, with or without lactophenol cotton blue staining.

\section{RESULTS AND DISCUSSION}

\section{Factors affecting arthroconidium formation in T. rubrum}

Arthroconidium formation occurred in all five isolates of T. rubrum used in preliminary studies, when $10 \%$ of the incubation atmosphere was replaced by $\mathrm{CO}_{2}$ on SDA media at $37^{\circ} \mathrm{C}$ after 10 days. These conditions were used as standard in the comparison of the effect of different factors on arthroconidium formation. When cultured in normal air, the frequency of arthroconidium formation was zero. Analysis of the time-course of arthroconidium production in T. rubrum N250 showed that optimal arthroconidium formation was at 10 days (Fig. 1), and the optimal percentage of $\mathrm{CO}_{2}$ for arthroconidium formation in T. rubrum N250 was $10 \%$ (Fig. 2). Arthroconidium production in T. mentagrophytes in vitro at $31^{\circ} \mathrm{C}$ is also induced by

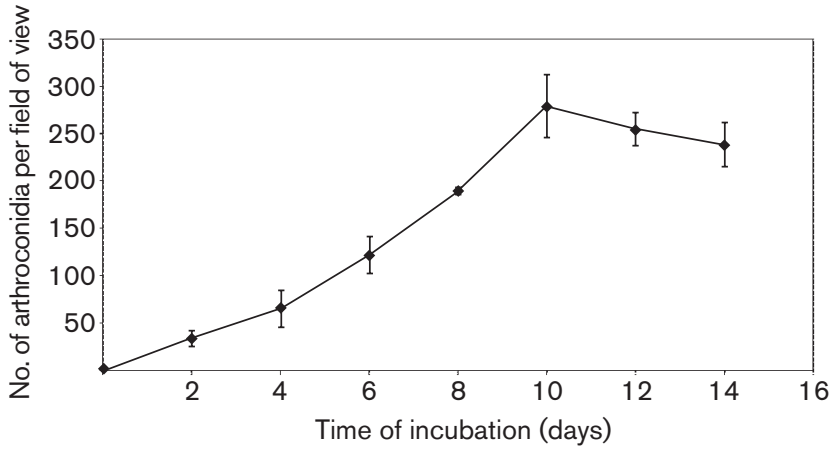

Fig. 1. Time-course of arthroconidia formation in T. rubrum $\mathrm{N} 250$ on SDA at $37{ }^{\circ} \mathrm{C}$ and $10 \% \mathrm{CO}_{2}$. Means and $95 \%$ confidence limits are shown $(n=3)$.

cultivation in an atmosphere with higher $\mathrm{CO}_{2}$ tension (Bibel et al., 1979). Curiously, Gupta et al. (2003) have found that Trichophyton raubitschekii, which some authors consider to be conspecific with T. rubrum, produces arthroconidia under normal aerobic conditions, and that this is not stimulated by $10 \% \mathrm{CO}_{2}$. Whether the stimulated arthroconidiogenesis is due to the presence of $\mathrm{CO}_{2}$ itself or reduced oxygen tension has been the subject of some controversy (Barrera, 1986). Some investigators (Emyanitoff \& Hashimoto, 1979) have shown that exogenous $\mathrm{CO}_{2}$ is not the absolute requirement, and that replacement of air by either $\mathrm{N}_{2}$ or $\mathrm{CO}_{2}$ stimulates arthrosporulation in T. mentagrophytes to the same extent. However, King et al. (1976) have shown that while helium and methane, like $\mathrm{CO}_{2}$, tend to inhibit the growth of $T$. mentagrophytes, they do not induce arthroconidial formation. $\mathrm{CO}_{2}$ diffuses physiologically from normal skin (Frame et al., 1972), and its level rises in damaged or occluded skin (Malten \& Thiele, 1973). Thus a rise in $\mathrm{CO}_{2}$ tension together with a reduction in oxgen tension during infection may cause the transformation of dermatophyte hyphae to arthroconidium chains (Allen \& King, 1978).

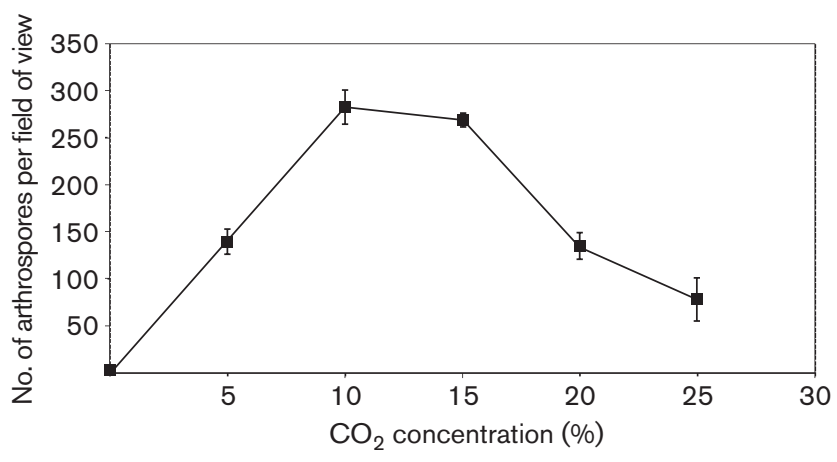

Fig. 2. Effect of $\mathrm{CO}_{2}$ concentration on arthroconidia formation in T. rubrum N250 after 10 days on SDA at $37^{\circ} \mathrm{C}$. Means and $95 \%$ confidence limits are shown $(n=3)$. 
Table 1. Effect of five different media on arthroconidial production in five strains of $T$. rubrum

The mean and $95 \%$ confidence limits are shown $(n=3)$.

\begin{tabular}{|c|c|c|c|c|c|}
\hline Strain & \multicolumn{5}{|c|}{ Medium } \\
\hline $\mathrm{N} 251$ & $75 \cdot 0 \pm 19 \cdot 7$ & $45 \cdot 0 \pm 10 \cdot 8$ & $269 \cdot 7 \pm 5 \cdot 2$ & $160 \cdot 0 \pm 41 \cdot 8$ & $115 \cdot 0 \pm 11 \cdot 4$ \\
\hline 15477 & $80 \cdot 0 \pm 13 \cdot 8$ & $59 \cdot 0 \pm 12 \cdot 4$ & $180 \cdot 0 \pm 4 \cdot 3$ & $130 \cdot 0 \pm 10 \cdot 8$ & $85 \cdot 0 \pm 23 \cdot 7$ \\
\hline 14686 & $77 \cdot 0 \pm 24 \cdot 4$ & $34 \cdot 0 \pm 13 \cdot 1$ & $160 \cdot 0 \pm 11 \cdot 4$ & $155 \cdot 0 \pm 11 \cdot 4$ & $150 \cdot 0 \pm 227$ \\
\hline
\end{tabular}

In the presence of $10 \% \mathrm{CO}_{2}$, SDA was the optimal medium for arthroconidium formation for most strains. No growth was seen in media containing $>3 \% \mathrm{NaCl}$ or $>3 \%$ peptone, and the addition of $1 \% \mathrm{NaCl}$ did not increase arthroconidium formation (Table 1). Levels of arthroconidium formation were reduced even further with other media. Miyazi \& Fujiwara (1971) have reported that T. rubrum produces a greater number of spherical cells (arthroconidia) on $\mathrm{BHI}$ at $37^{\circ} \mathrm{C}$ than on other media, and that the presence of $0.85 \% \mathrm{NaCl}$ or ornithine is important for arthrosporulation.

When cultures were incubated at various temperatures with $10 \% \mathrm{CO}_{2}$, a temperature of $37^{\circ} \mathrm{C}$ was found to be maximal for arthroconidium formation (Fig. 3). At $42{ }^{\circ} \mathrm{C}$, no growth occurred. Temperatures between 32 and $39^{\circ} \mathrm{C}$ also strongly favour the arthroconidiation of T. mentagrophytes on SDA, and no or less arthroconidium formation is seen in $T$. mentagrophytes at $30^{\circ} \mathrm{C}$ (Emyanitoff \& Hashimoto, 1979); in addition, arthroconidium formation in T. rubrum was seen at $37^{\circ} \mathrm{C}$ but not at $25^{\circ} \mathrm{C}$ by Miyazi \& Nishimura (1971). The temperature required for optimal arthroconidium formation in vitro, $37^{\circ} \mathrm{C}$, is higher than those recorded for the surface of the human body, typically $33 \pm 1{ }^{\circ} \mathrm{C}$, and near the upper limit for growth for this species. Although, generally, slightly acidic conditions favoured arthrosporulation, the optimal $\mathrm{pH}$ for arthroconidium formation was

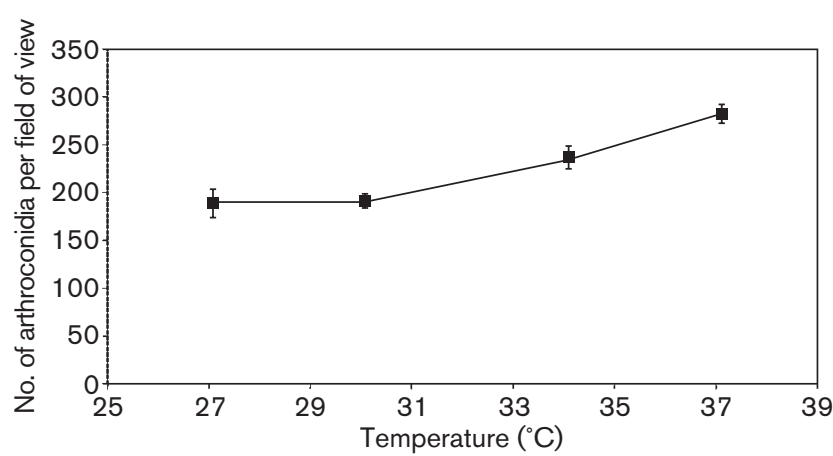

Fig. 3. Effect of temperature on arthroconidia formation in $T$. rubrum $\mathrm{N} 250$ after 10 days on SDA at $37^{\circ} \mathrm{C}$ and $10 \% \mathrm{CO}_{2}$. Means and $95 \%$ confidence limits are shown $(n=3)$. found to be $7 \cdot 5$ (Fig. 4). The optimal $\mathrm{pH}$ of the medium for arthroconidium formation in T. rubrum was found to be $7 \cdot 4$ by Miyazi et al. (1971).

\section{Effect of antifungal agents and betamethasone on arthroconidia formation}

Subinhibitory levels of several antifungal agents were tested for their effects on arthroconidium formation on SDA at $37^{\circ} \mathrm{C}$. At subinhibitory concentrations, some of these antifungal agents, including amphotericin B, griseofulvin and clotrimazole, stimulated the level of arthrosporulation, but itraconazole, and particularly terbinafine, suppressed arthroconidium production (Table 2). Similar effects have been seen in T. mentagrophytes, in which griseofulvin $\left(0 \cdot 5 \mu \mathrm{g} \mathrm{ml}^{-1}\right)$, clotrimazole $\left(0 \cdot 1 \mu \mathrm{g} \mathrm{ml}^{-1}\right)$, and particularly amphotericin B $\left(5 \mu \mathrm{g} \mathrm{ml}^{-1}\right)$, stimulate a low level of arthrosporulation (Emyanitoff \& Hashimoto, 1979). Compared to other antifungals, itraconazole, and particularly terbinafine, have significantly increased rates of cure and shorter treatment times (Roberts, 1999), and interestingly, in our study, only itraconazole, and particularly terbinafine, suppressed arthroconidium production in $T$. rubrum N250. These results may be clinically significant: if sufficiently fungicidal concentrations are not attained in some areas of the lesion, some antifungals may enhance the conversion of hyphae to arthroconidia. Hashimoto \&

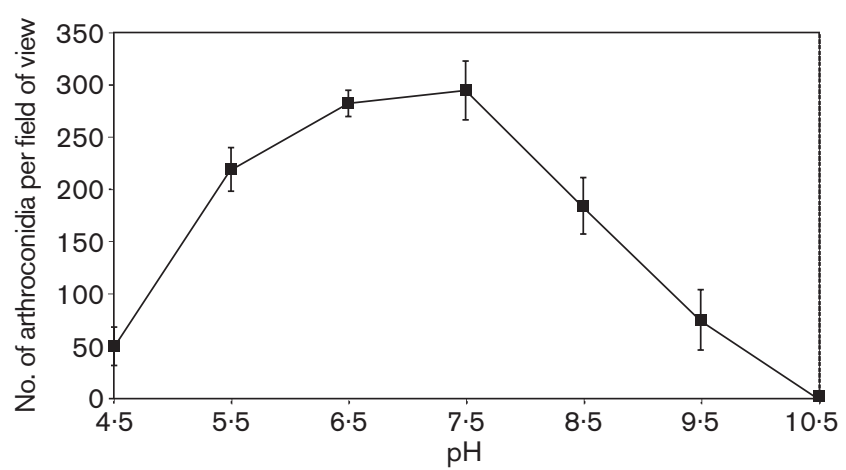

Fig. 4. Effect of $\mathrm{pH}$ on arthroconidia formation in T. rubrum $\mathrm{N} 250$ after 10 days on SDA at $37^{\circ} \mathrm{C}$ and $10 \% \mathrm{CO}_{2}$. Means and $95 \%$ confidence limits are shown $(n=3)$. 
Table 2. Effect of different antifungals on T. rubrum N250

NA, Not applicable.

\begin{tabular}{|lcc|}
\hline Antifungal & $\begin{array}{c}\text { Concentration } \\
\left(\boldsymbol{\mu} \mathbf{~ I ~}^{-1}\right)\end{array}$ & $\begin{array}{c}\text { Mean no. } \\
\text { arthroconidia } \\
\text { per field of view }\end{array}$ \\
\hline Amphotericin B & $1 \cdot 0$ & $395 \pm 5 \cdot 4$ \\
Griseofulvin & $5 \cdot 0$ & $340 \pm 9 \cdot 3$ \\
Clotrimazole & $0 \cdot 25$ & $364 \pm 11 \cdot 3$ \\
& $0 \cdot 5$ & $295 \pm 14 \cdot 2$ \\
Itraconazole & $0 \cdot 1$ & $373 \pm 6 \cdot 1$ \\
& $0 \cdot 5$ & $314 \pm 8 \cdot 8$ \\
Terbinafine & $0 \cdot 25$ & $210 \pm 10 \cdot 1$ \\
& $0 \cdot 5$ & $175 \pm 17 \cdot 7$ \\
DMSO (10\%) & $0 \cdot 001$ & $120 \pm 8 \cdot 1$ \\
Sabouraud broth control & $0 \cdot 05$ & $95 \pm 7 \cdot 3$ \\
& $\mathrm{NA}$ & $275 \pm 19 \cdot 3$ \\
& $\mathrm{NA}$ & $280 \pm 5 \cdot 4$ \\
\hline
\end{tabular}

${ }^{*}$ The mean and $95 \%$ confidence limits are shown $(n=3)$.

Blumenthal (1978) have reported that dormant arthroconidia of $T$. mentagrophytes are resistant to common antimycotics such as griseofulvin, clotrimazole, miconazole and nystatin. This resistance may be due to the nature of the arthroconidial wall. The steroid betamethasone 17-valerate at $1 \mathrm{mg} \mathrm{ml}^{-1}$ caused appreciable stimulation of arthroconidium formation $(352 \pm 2.6$ arthoconidia per field of view) compared to the control $(255 \pm 2 \cdot 2)$ (mean of three experiments $\pm \mathrm{SD}$ ). An early report (Chattaway et al., 1959) showed an inhibition of the growth of T. rubrum by different steroids. Thus, steroids may influence either directly or indirectly the development of dermatophyte infections.

\section{Clinical isolates of $T$. rubrum}

Of a total of 50 clinical isolates, obtained before, during and after treatment with itraconazole and terbinafine, only two strains were unable to produce arthroconidia under the previously defined optimal conditions. Of these isolates, 45 were from nine patients (two to six per patient), and therefore not necessarily independent isolates. However, the two isolates that failed to produce arthroconidia came from patients from whom arthroconidium-producing isolates had also been obtained. This might represent the suppression of the ability to produce arthroconidia in a strain during treatment or might represent different strains, since most toenail infections caused by T. rubrum have recently been shown to be caused by more than one strain (Yazdanparast et al., 2003). Fujita et al. (1986) found that two of eight strains of T. mentagrophytes did not produce arthroconidia; also, eight of 15 strains of T. rubrum examined by Miyazi \& Nishimura (1971) failed to produce arthroconidia. This discrepancy may relate to differences in methodology.

\section{Arthroconidial formation in ex vivo nails}

Arthroconidial production in ground nails from the toenails of volunteers occurred under the same optimal conditions, and again did not occur unless the atmosphere was replaced with $10 \% \mathrm{CO}_{2}$, although it took longer (14 days) to reach maximal levels. This suggests that during invasion of the nail by hyphal growth, changes to the environment of the fungus caused by the reaction of the host tissue to the fungus induce the formation of arthroconidia. For example, hyperkeratosis, often seen in onychomycosis, may lead to a decrease in local $\mathrm{O}_{2}$ concentration and/or increase in $\mathrm{CO}_{2}$ concentration, as seen in damaged skin (Malten \& Thiele, 1973). It has recently been demonstrated in a similar nail model (though without arthroconidial formation) that the mimimum fungicidal concentration of terbinafine against T. rubrum within nails is increased up to 100 -fold compared with standard media (Osborne et al., 2004). This suggests, in general, that the properties of this dermatophyte in nail models are likely to differ significantly from those of fungi grown on laboratory media and to be more representative of the situation in vivo.

It has yet to be demonstrated that arthroconidia of $T$. rubrum formed in vitro, and therefore presumably in vivo, are more resistant to antifungal activity than hyphae. Terbinafine, as one of the main therapeutic agents for onychomycosis, has been demonstrated to be fungicidal, though this has only been shown using actively growing hyphae (Hazen, 1998). The thick walls of arthroconidia may resist antifungal treatment, only to germinate following the cessation of treatment. Indeed, an approach designed to stimulate the germination of resistant propagules in onychomycosis patients who failed antifungal therapy by applying slides of Sabouraud agar to diseased nails for $24 \mathrm{~h}$ prior to topical antifungal treatment has been shown to have a high cure rate (Pierard et al., 2000).

In conclusion, this study has shown how certain conditions, including physiological changes within the nail and antifungal agents, may trigger the formation of arthroconidia from hyphal cells, and that a key factor is the presence of $10 \% \mathrm{CO}_{2}$. Further work is needed to examine the widely assumed importance of the role of arthroconidia in pathogenesis and the resistance of dermatophytes to effective antifungal treatment.

\section{REFERENCES}

Allen, A. M. \& King, R. D. (1978). Occlusion, carbon dioxide and fungal skin infections. Lancet 1, 360-362.

Aly, R. (1994). Ecology and epidemiology of dermatophyte infections. J Am Acad Dermatol 31, S21-S25.

Barrera, C. R. (1986). Formation and germination of fungal arthroconidia. CRC Crit Rev Microbiol 12, 271-292.

Bibel, D. J., Crumrine, D. A., Yee, K. \& King, R. D. (1979). Development of arthrospores of Trichophyton mentagrophytes. Infect Immun 15, 958-971. 
Chattaway, F. W., Townsley, J. D. \& Barlow, A. J. E. (1959). Effect of steroids and related compounds on the growth of dermatophytes. Nature 184, 1731.

Chin, B. \& Knight, S. G. (1957). Growth of Trichophyton mentagrophytes and Trichophyton rubrum in increased carbon dioxide tensions. J Gen Microbiol 16, 642-646.

Emyanitoff, R. G. \& Hashimoto, T. (1979). The effects of temperature, incubation atmosphere, and medium composition on arthrospore formation in the fungus Trichophyton mentagrophytess. Can J Microbiol 25, 362-366.

Evans, E. G. V. (1999). Causative pathogens in onychomycosis and the possibility of treatment resistance: a review. J Am Acad Dermatol 38, S32-S36.

Evans, E. G. V. \& Roberts, D. T. (1998). Subungual dermatophytoma complicating dermatophyte onychomycosis. British Association of Dermatologists. Br J Dermatol 138, 189-203.

Frame, G. W., Straus, W. G. \& Mailbach, H. I. (1972). Carbon dioxide emission of the human arm and hand. J Invest Dermatol 59, 155.

Fujita, S., Matsuyama, T. \& Sato, Y. (1986). A simple and reliable culturing method for production of arthroconidia by dermatophytes. Jpn J Med Mycol 27, 175-181.

Gupta, A. K., Ahmad, I., Porretta, M. \& Summerbell, R. C. (2003). Arthroconidial formation in Trichophyton raubitschekii. Mycoses 46, 322-328.

Hashimoto, T. \& Blumenthal, H. J. (1978). Survival and resistance of Trichophyton mentagrophytes arthrospores. Appl Environ Microbiol 35, 274-277.

Hazen, K. C. (1998). Fungicidal versus fungistatic activity of terbinafine and itraconazole: an in vitro comparison. J Am Acad Dermatol 38, S37-S41.

King, R. D., Dillavou, C. L., Greenberg, J. H., Jeppsen, J. C. \& Jaeger, J. S. (1976). Identification of carbon dioxide as a dermatophyte inhibitory factor produced by Candida albicans. Can J Microbiol 22, 1720 .

Malten, K. E. \& Thiele, F. A. J. (1973). Evaluation of skin damage. II - water loss and carbon dioxide release measurements related to skin resistance measurements. Br J Dermatol 89, 565-569.

Matsumoto, T. (1996). Fungal diseases in dermatology. In Principles and Practice of Clinical Mycology, pp. 105-129. Edited by C. C. Kibbler, D. W. R. Mackenzie \& F. C. Odds. Chichester: Wiley.

Miyaji, M. \& Fujiwara, K. (1971). Effect of amino acids, salt, and $\mathrm{pH}$ on the spherical cell formation. Jpn J Med Mycol 12, 200-205.

Miyazi, M. \& Nishimura, K. (1971). Studies on arthrospore of $T$. rubrum. Jpn J Med Mycol 12, 18-23.

Miyazi, M., Nishimura, K. \& Kariya, H. (1971). Relationship between the types of eruption and the parasitic form of T. rubrum. Jpn J Med Mycol 12, 81-85.

Osborne, C. S., Leitner, I., Favre, B. \& Ryder, N. S. (2004). Antifungal drug response in an in vitro model of dermatophyte nail infection. Med Mycol 42, 159-163.

Pierard, G. E., Pierard-Franchimont, C. \& Arrese, J. E. (2000). The boosted antifungal topical treatment (BATT) for onychomycosis. Med Mycol 38, 319-392.

Roberts, D. T. (1999). Onychomycosis: current treatment and future challenges. Br J Dermatol 141 (Suppl. 56), 1-4.

Weigl, E. \& Hejtmanek, M. (1979). Differentiation of Trichophyton mentagrophytes arthrospores controlled by physical factors. Mykosen 22, 167-172.

Wright, L. R., Scott, E. M. \& Gorman, S. P. (1984). Spore differentiation in a clinical strain of Trichophyton mentagrophytes. Microbios 39, 87-93.

Yazdanparast, A., Jackson, C. J., Barton, R. C. \& Evans, E. G. V. (2003). Molecular strain typing of Trichophyton rubrum indicates multiple strain involvement in onychomycosis. Br J Dermatol 148, 51-54. 